\title{
MAC protocol requirements for OFDMA-PONs
}

\author{
Wansu Lim, Milos Milosavljevic, Ali Gliwan, Pandelis Kourtessis, John M. Senior \\ Optical Networks Group, Science and Technology Research Institute (STRI), \\ University of Hertfordshire College Lane Campus, Hatfield, AL10 9AB, UK \\ Tel: +44 (0) 1707285082,e-mail:w.lim9@herts.ac.uk
}

\begin{abstract}
This paper provides specifications of the parameters defining the design of medium access control (MAC) protocols for orthogonal frequency division multiple access (OFDMA) passive optical networks (PONs). A Dynamic bandwidth allocation (DBA) algorithm exhibiting service level agreement (SLA) has displayed packet delays of less than $1.5 \mathrm{~ms}$ for the middle and high SLA optical network units (ONUs) at $156 \mathrm{Mbps}$, representing the maximum ONU offered load. A spectral efficiency of $93.75 \%$ the total network capacity of 40 Gbps has been achieved, for 256 ONUs and long-reach link lengths of $100 \mathrm{~km}$. The new protocol functionalities have been attained by the extension of the XG-PON frame structure to demonstrate OFDMA/TDMA granularity.
\end{abstract}

Keywords: Next generation access networks, orthogonal frequency division multiplexing (OFDM), passive optical networks (PONs) and medium access control (MAC) protocol.

\section{INTRODUCTION}

Orthogonal frequency division multiple access (OFDMA) Passive Optical Networks (PONs) have been gaining in capacity, reach and cost [1], in response to the targets of next generation access networks. Consolidating the requirements in service provisioning, including Ultra High Definition Television (UHDTV), 3-Dimensional TV (3DTV) and business E-Line Point-to-Point (P2P) to mention a few, the guaranteed bit rates of residential users and small businesses are expected to grow symmetrically to $400 \mathrm{Mbps}$ [2]. In addition corporations and telco services require bandwidths of several tens of Gbps while base stations for long term evolution advanced (LTEA) backhauling up to 3 Gbps per site [3]. To attend to these requirements, the FP7 project ACCORDANCE [4] aims, besides the physical layer, to develop new medium access control (MAC) designs for OFDMA-PONs.

Among the OFDMA-PON protocol proposals, the algorithm in [5] uses fixed subcarriers for control signalling and hybrid subcarrier/time-slots for data transfer. Frequent communication of report messages every $10.5 \mu \mathrm{s}$ allows accurate specification of optical network unit (ONU) queues, but at the expense of intensive processing in the optical line terminal (OLT) and high occupancy of the upstream bandwidth. PONIARD [6] portrays hybrid OFDMA/TDMA allocation but with pure TDMA used extensively. In [7] a dynamic circuit transmission (DCT) protocol is presented with three-way signalling for service connectivity. DCT could result in increased queuing delays, especially for short-lived circuits, while being intolerant to rapidly fluctuating traffic.

Dynamic bandwidth allocation (DBA) in ACCORDANCE is optimized by exploring OFDMA/TDMA scheduling at both ONU and network segment levels, accounting for issues such as service level agreement (SLA), limited subcarrier availability and/or ONU bandwidth granularity to less than 300-400 Mbps, potentially available with every subcarrier. To exploit the spectral efficiency characteristics of OFDMA, adaptive subcarrier modulation and cross layer optimization are also supported in the new frame formats.

\section{OFDMA-PON MAC CRITERIA}

\subsection{Network Topology, ONU and connectivity types}

The ACCORDANCE architecture is shown in Fig. 1, exploiting the merits of OFDMA in scaling up the access network in reach and aggregate rates while achieving convergence of technologies [4]. This paper includes a brief account of its fundamental operation but it mainly focuses on ONU consolidation and connectivity types and their contribution on defining the OFDMA MAC. The ACCORDANCE infrastructure exploits the transmission of subcarriers to/from ONUs through a single feeder fiber between the OLT and a remote node (RN). This RN, shown in Fig. 1 at the first splitting point ("Level 1 RN"), employs a power coupler/splitter, potentially also using a wavelength selective device to reduce splitting losses. ONUs can be located at user premises (FTTH or FTTB via DSL MDU/MTU), wireless base stations, or legacy PON OLT/DSLAMs. The subcarriers are grouped to form FDM channels (network segments) for carrying traffic belonging to all deployed services or assigned to specific service clusters according to operator needs.

In common with TDMA protocols, the network reach, ONU number and upstream (US) bitrate are parameters that influence the protocol design and DBA algorithm. In addition, in OFDMA-PONs the number of subcarriers, the downstream (DS) as well as US bitrate, the RN implementation and the US/DS modulation formats are also significant. The latter defines a set of cross-layer requirements for the MAC since the signal-tonoise ratio (SNR) of supported subcarriers specifies their spectral efficiency. This is turn classifies the overall capacity of subcarrier clusters and their ability to satisfy the bandwidth requirement of retrospective ONUs. In view of an arrayed waveguide grating (AWG) at Level $1 \mathrm{RN}$, different wavelengths are routed to different parts of the network, targeting different ONUs and the MAC incorporates a third level of multiplexing to assign 
subcarriers to more than one $(1: 4,1: 8)$ supported wavelengths. The selective assignment of the OFDMA spectrum leads to inter-segment bandwidth allocation. To accommodate the variety of ONU services in the ACCORDANCE OFDMA-PON the protocol design needs to exhibit flexible modes of subcarrier allocation, achieved by hybrid algorithms imposing granularity and service optimisation. Quality of Service (QoS) is implemented in the devised algorithms by the provision of SLA and Class of Service (CoS) differentiation.

The ONU types terminated to the network OLT in ACCORDANCE represent different technologies. OFDMA terminations, legacy Ethernet/Gigabit PONs (E/GPONs), their updates and wireless/mobile ONU define distinctive requirements in data transport and bandwidth and as a result the assignment of ONUs to SLA and CoS. With respect to the protocol itself, new OFDMA frame formats need to be developed that allow hybrid subcarrier and time operation and have originated from legacy PON frame structures.

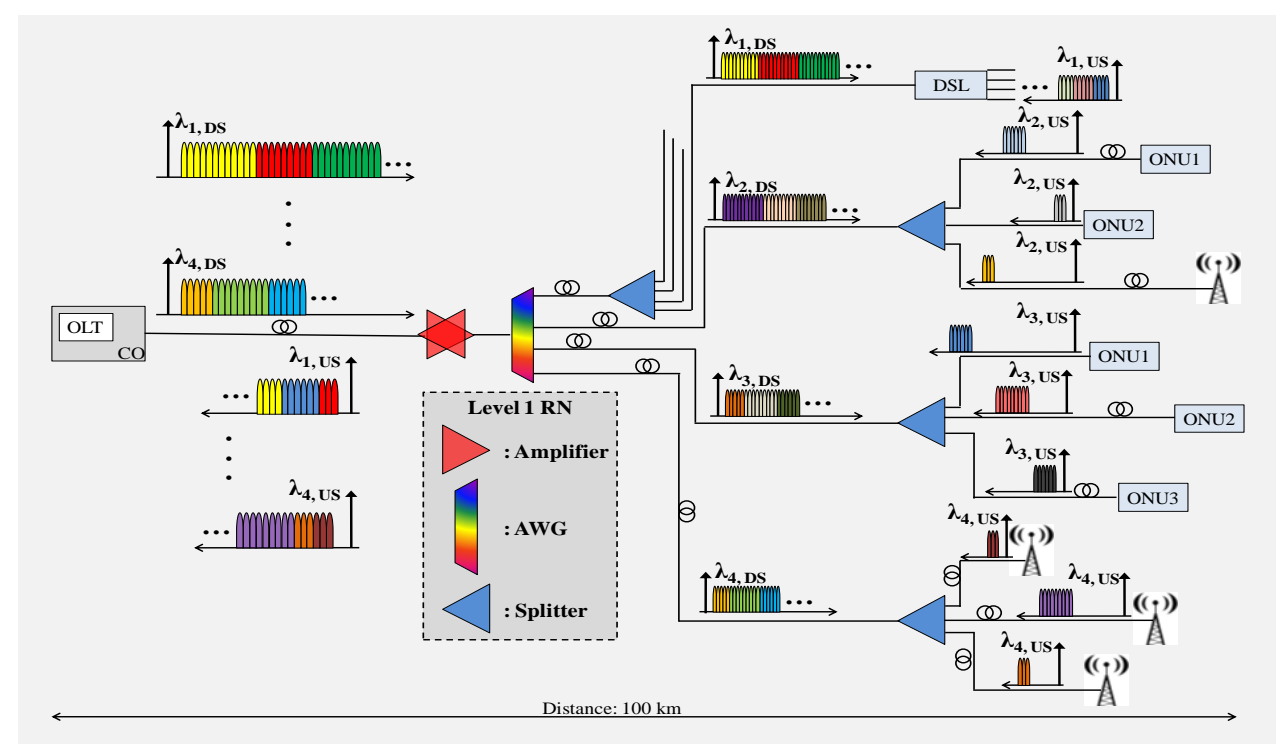

Figure 1. The ACCORDANCE Network.

\subsection{OFDMA-PON deployment scenarios}

A number of network scenarios have been promoted in ACCORDANCE for OFDMA-PON performance evaluation. The network reach is naturally vital since longer-reach implementations require enhanced MAC efficiency given the larger propagation delays and perhaps increased differential delays among ONUs. The overall performance of the OFDMA/TDMA MAC over the optical backhaul in terms of packet delays should also conform to the requirements of LTE for successful wireless convergence. The correlation between the number of ONUs and subcarriers largely affects the MAC algorithm mode of operation. Bandwidth assignment only in the subcarrier domain cannot be possible if the number of subcarriers is less than the number of supported ONUs. Subcarrier availability in practise can be limited by constraints on digital signal processors, imposed by high computation complexity. Therefore hybrid OFDMA/TDMA solutions must be employed to share subcarriers between ONUs in time, providing also for finer granularity of bandwidth.

Exploiting the above recommendations, the urban scenario assumes a completely passive, $20 \mathrm{~km} \mathrm{reach}$, access network for densely populated areas. A high-splitting ratio of 256 ONUs per wavelength is the target with an overall capacity of $100 \mathrm{Gbps}$ per wavelength, providing a guaranteed bit rate of $400 \mathrm{Mbps}$ per residential user. Scenarios involving fewer ONUs could also be considered to accommodate higher bandwidth requests per ONU as it would be the case for example in the presence of ONU/BSs.

The passive extended reach scenario increases the network span to $40 \mathrm{~km}$ without amplification at the Outside Plant (OSP), targeting network consolidation in metro-regional areas as well as wider coverage for rural areas. The supported ONUs are expected to be decreased, offering higher bandwidth per ONU (625 Mbps for 40 Gbps aggregate capacity per wavelength) through a 1:64 splitting ratio, maintaining subcarrier numbers to 256.

Finally the Active Extended Reach scenario targets rural and special network consolidation architectures. Signal amplification is expected to be performed at the Level $1 \mathrm{RN}$ site allowing for the maximum reach of 100 $\mathrm{km}$. However, depending on the exact deployment specifications, it could take place at a remote Central office (CO). This scenario is expected to draw quite diverse packet delay and network throughput figures compared to any other implementation and best suited to explore network migration scenarios.

\section{HYBRID OFDMA/TDMA protocol design}

This section describes the Sequential Dynamic Subcarrier Assignment (SDSCA) protocol design implemented for ACCORDANCE. The SDSCA operation assumes non-status-reporting ONUs, by means of a Monitoring Mechanism, utilizing a monitoring window and grant messages as shown in [8] for bandwidth allocation. The 
developed simulation design in OPNET exhibits a $100 \mathrm{~km}$ OFDMA-PON composed of one OLT and 256 ONUs. Three SLAs, SLAt, $\mathrm{t}=0,1,2$, from high to low priority have been considered. The number of ONUs in each service level is set to 16,80 and 160. The total upstream data capacity is $40 \mathrm{Gbps}$, arranged in 256 subcarriers of 156.25 Mbps each. Grant processing and propagation delays are considered as $0.5 \mu \mathrm{s}$ and $0.5 \mu \mathrm{s} / \mathrm{km}$ respectively. The network traffic is implemented by a Pareto self-similar traffic model with a typical Hurst parameter of 0.8 to simulate practical network patterns. The packet size is uniformly generated between 64-1518 Bytes.

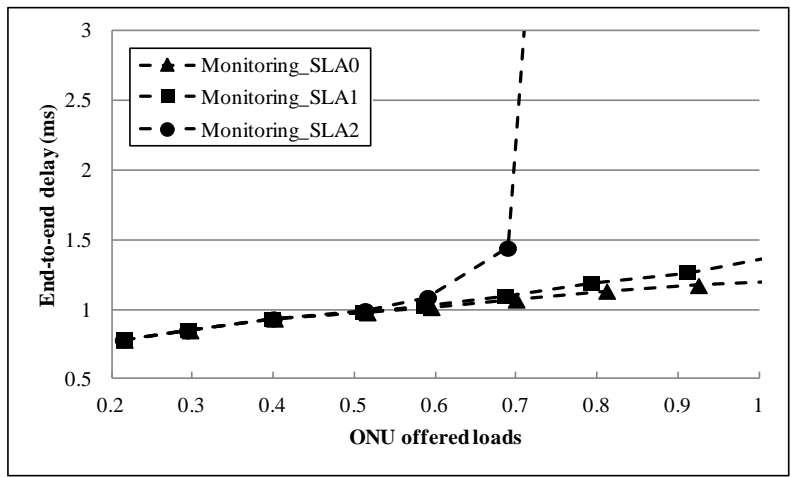

(a)

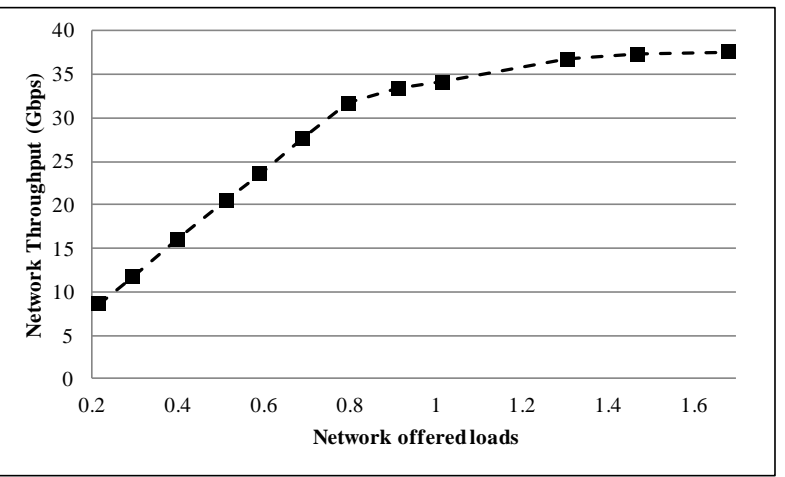

(b)

Figure 2. (a) End-to-end packet delay, (b) Network throughput for a $100 \mathrm{~km}$, long-reach OFDMA-PON.

Shown in Fig. 2 (a) the end-to-end packet delay of the low priority SLA, SLA2, saturates at 0.7 offered load, with the middle and high priority SLAs displaying less than $1.5 \mathrm{~ms}$ packet delay, even if the ONU offered load is 1.0. Figure 2 (b) displays the network performance in throughput in the presence of 256 ONUs. A network load of 1.0 corresponds to $40 \mathrm{Gbps}$ data rates. The SDSCA algorithm manages to utilize $93.75 \%$ of the total capacity. Although the network reach extends to $100 \mathrm{~km}$, the performance figures demonstrate high efficiency due to the application of non-status-reporting and its capability to allocate ONU bandwidth independently of the exchange of report messages. This makes non-status reporting particularly attractive for long-reach DBA applications.

\section{MAC frame functionalities and control fields}

Bearing in mind that the specifications of the OFDMA MAC should provide an evolutionary solution for existing protocols, this section defines the modifications imposed on the XGPON standard, G.987.3 [9] to accommodate the requirements for downstream and upstream transmission of OFDMA-PONs.
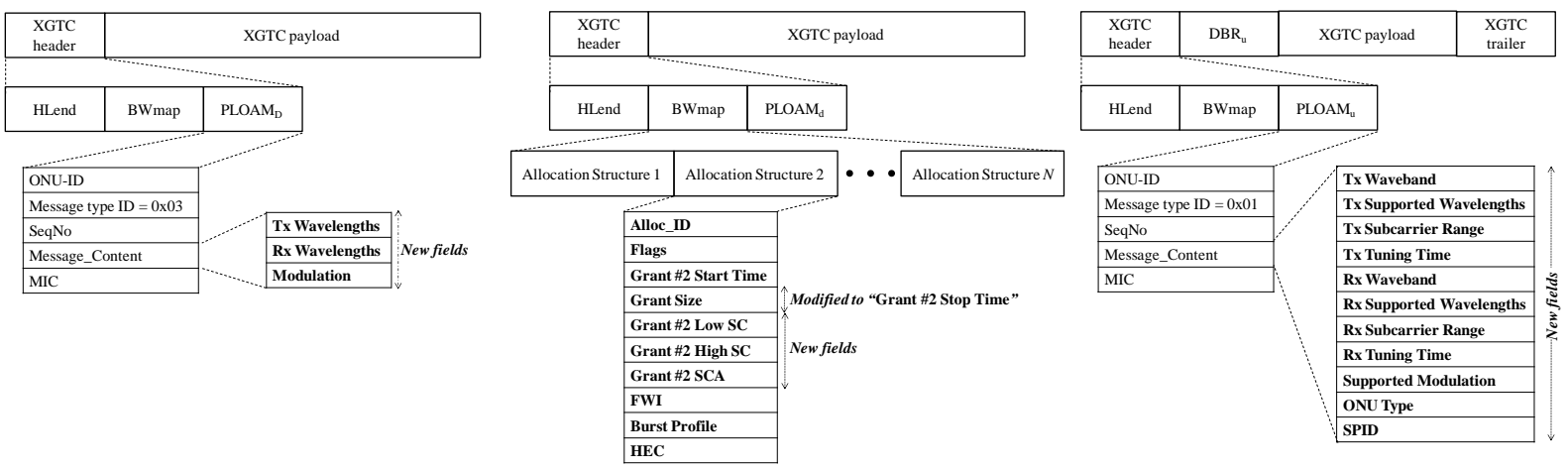

Figure 3. (a) Modified Assign_ONU-ID message, (b) Modified US BW map in DS frames, (c) Modified Serial_Number_ONU message in XG-PON US frames.

\subsection{MAC Frame functionalities}

The ACCORDANCE US frame integrates fields such as the $O N U_{-} I D$ and $C o S$, the $O N U_{-} L S C_{-} I D$ and $O N U_{-} H S C_{-} I D$, defining the start and end of subcarrier ranges, the service provider identifier, $S P \_I D$, field allowing for multiple providers' support and the ONU_Type field to identify the various ONU types. The Sup_Mod field is also included to define an ONU's QAM efficiency for adaptive subcarrier modulation.

New to the DS frame header is the SubCarrier Allocation identifier, SCA, field used by the OLT to distinguish between the bandwidth allocation mode. The Low_SC and High_SC fields, alongside the LSC_Start_Time and HSC_Start_Time fields will be explained in the section below.

\subsection{XGPON upgrade for OFDMA operation}

During registration in the XG-PON standard the OLT sends registration messages to all ONUs using the PLOAM field of the downstream frame. The "Serial_Number_ONU" message is contained in the Message type 
ID field with number 0x03, as shown in Fig. 3 (a). To accommodate the defined features the Message_Content field of the downstream PLOAM XG-PON frame (empty in the standard) has been modified.

Additionally a new message type is introduced inside the XG-PON PLOAMd downstream field, under Message Type ID field with number 0x13, named "Rx_Config". This is used to allow the OLT define by means of the Low_SC and High_SC the subcarrier range used by each downstream ONU. To accommodate adaptive modulation, new message types are necessary to be included in the downstream PLOAM field (under Message Type ID field with numbers $0 \times 14$ and 0x15). The proposed message types are represented by the "Adaptive_Modulation" and "Req_BER Monitor" messages. In the former the OLT instructs ONUs to adjust their receiver to a specific QAM modulation format. In the latter it requests ONUs to monitor downstream BER.

To accommodate OFDMA/TDMA functionality, the US grant messaging of the XG-PON standard also needs modification, as depicted in Fig. 3 (b). Originally the grant messages of XG-PON lie inside the XGTC header, under the BWmap field. XG-PON utilises the Grant Start Time and Grant Size sub-fields for bandwidth allocation (\#2 defines the ONU used here as an example). To that extent, the Grant Size sub-field has been modified to a Grant Stop Time sub-field as shown in Fig. 3 (b). In addition, extra sub-fields are incorporated in the $B W m a p$ to define, apart from the start and stop times of each subcarrier, also the first and last subcarriers in the supported spectrum for each ONU.

Subsequently US frame modifications are also critical for the new protocol operation. The XG-PON upstream PLOAM already defines a "Serial_Number_ONU" message signified by the message ID 0x01. To include the additional fields required by the OFDMA protocol in communicating ONU capabilities to the OLT, the Message_Content field has been appropriately modified as shown in Fig. 3 (c). For the purpose of reporting the bandwidth requests and actual transmission of data from ONUs, the "Report" message of the XG-PON standard is used. To enable adaptive subcarrier assignment, the "Acknowledge" message of the XG-PON US PLOAM is modified. The corresponding Message_Content field is extended following the recommendations already presented for DS.

\section{CONCLUSIONS}

With the scope of providing recommendations for original MAC designs for OFDMA-PONs, the ACCORDANCE network features imposing an impact on the potential network MAC topologies and bandwidth allocation schemes have been presented. These distinguish between network dimensioning parameters (network reach, subcarriers and ONUs number, the Upstream/Downstream Bitrates), the US/DS modulation schemes for increased spectral effciency and the bandwidth allocation criteria. Long reach networks have also been evaluated for increased ONU numbers reaching up to 256 under a distance of $100 \mathrm{~km}$, demonstrating high throughput and low packet-delay figures. The intended protocol functionalities with respect to subcarrier adaptive modulation and hybrid OFDMA/TDMA operation constitute the provision of the network MAC for dynamic bandwidth allocation. To that extend the requirements of the new frame control fields have been defined, based on the XGPON frame format, allowing in ACCORDANCE for distinctive modes of bandwidth allocation.

\section{ACKNOWLEDGEMENTS}

This work was supported by the ACCORDANCE project, through the 7th ICT Framework Programme.

\section{REFERENCES}

[1] N. Cvijetic et al., "Orthogonal frequency division multiple access PON (OFDMA-PON) for colorless upstream transmission beyond $10 \mathrm{~Gb} / \mathrm{s}$," IEEE J. Sel. Areas Commun., vol. 28, pp. 781-790, Aug. 2010.

[2] J.-I. Kani et al., "Next-Generation PON—Part I: Technology Roadmap and General Requirements," IEEE Commun. Mag., vol. 47, no. 11, pp. 43-49, Nov. 2009.

[3] A. Ghosh et al., "LTE-advanced: next-generation wireless broadband technology," IEEE Wireless Commun., vol. 17, pp. 10-22, June 2010.

[4] EU FP7 Project, ACCORDANCE, (A Converged Copper-Optical-Radio OFDMA-based access Network with high Capacity and Flexibility), Available: www.ict-accordance.eu

[5] J. Zhang et al., "An Efficient MAC Protocol for Asynchronous ONUs in OFDMA PONs," in Proc. OSA OFC 2011, Mar. 2011.

[6] W. Wei et al., "PONIARD: A Programmable Optical Networking Infrastructure for Advanced Research \& Development of Future Internet," J. Lightwave Technol., vol. 27, no. 3, pp. 233-241, Feb. 2009.

[7] W. Wei et al., "MAC protocols for optical orthogonal frequency division multiple access (OFDMA)-based passive optical networks," in Proc. OFC 2008, Mar. 2008.

[8] W. Lim et al., "QoS Aware MAC Protocol for OFDMA-PON," in Proc. ICTON 2011, pp. Tu.B6.1.

[9] ITU-T, "G.987.3 10-Gigabit-capable passive optical networks (XG-PON): Transmission convergence (TC) specifications" ITU-T 2010. 